\title{
Singular-perturbative Reduction to Birkhoff Normal Form and Instanton-Type Formal Solutions of Hamiltonian Systems
}

\author{
To the memory of the late Professor Nobuhisa Iwasaki
}

By

Yoshitsugu TAKEI*

\section{$\S 1$. Introduction}

As is illustrated by the computation of monodromy groups of second-order Fuchsian equations (cf. [AKT1]), the exact WKB analysis provides us with a powerful tool for studying global behavior of solutions of linear ordinary differential equations. To generalize such an analysis to nonlinear equations, T. Kawai (RIMS, Kyoto Univ.), T. Aoki (Kinki Univ.) and the author have developed the WKB theory for Painleve equations with a large parameter in our series of articles ([KT1], [AKT2], [KT2]). (See [T1], [T2] also.) In our treatment 2-parameter formal solutions called instanton-type solutions, which were constructed through the multiple-scale analysis in [AKT2], are playing a central role. Although we have succeeded in analyzing their local structure near simple turning points in [KT2], some of their important properties such as the behavior near fixed singular points have not been clarified yet. In this paper, to investigate their behavior near fixed regular-type singular points, we propose a new construction of 2-parameter formal solutions of Painlevé equations with a large parameter.

The new construction of formal solutions we propose here is based on Takano's work [Tka1] (see [Tka2] also), where he constructed a 2-parameter family of analytic solutions at each regular-type singular point of (ordinary) Painlevé equations. He made use of the well-known fact that Painlevé equations can be written in the form of Hamiltonian systems (which we call Painlevé Hamiltonian systems here) and established some reduction theorem for

Received July 31, 1998.

1991 Mathematics Subject Classification(s): 34E15, 58F05, 58F36

* Research Institute for Mathematical Sciences, Kyoto University, Kyoto 606-8502, Japan. 
Hamiltonian systems to construct analytic solutions. His reduction theorem is closely related to the following "Birkhoff normal form" of Hamiltonian systems (cf. [B], [SM]).

Birkhoff normal form. Consider a Hamiltonian system

$$
d q / d t=\partial H / \partial p, \quad d p / d t=-\partial H / \partial q
$$

with a Hamiltonian $H=H(t, q, p)$. If we can find a canonical transformation $(q, p) \rightarrow(\tilde{q}, \tilde{p})$ which transforms the original system (1) to

$$
d \tilde{q} / d t=\partial \tilde{H} / \partial \tilde{p}, \quad d \tilde{p} / d t=-\partial \tilde{H} / \partial \tilde{q}
$$

with

$$
\tilde{H}(t, \tilde{q}, \tilde{p})=\sum_{n \geq 0} \tilde{h}_{n}(t)(\tilde{q} \tilde{p})^{n+1}
$$

(i.e., $\tilde{H}$ is a function of $t$ and the product $\tilde{q} \tilde{p}$ only), then the new system (2) is called Birkhoff normal form of (1).

Roughly speaking, to construct 2-parameter formal solutions, we will modify Takano's proof so that it may be adapted to Hamiltonian systems of singular perturbations and prove the existence of a canonical transformation which reduces the Painlevé Hamiltonian system to its Birkhoff normal form in a singular-perturbative manner. The existence of singular-perturbative reduction will be shown in Section 2. Compared with the multiple-scale analysis employed in [AKT2], the method discussed in this paper is more singular-perturbative in the sense that a canonical transformation reducing to Birkhoff normal form can be determined recursively in an algebraic manner, i.e., solving differential equations degree by degree is not necessary. This character of the method actually enables us to analyze the behavior of our 2-parameter formal solutions near fixed regular-type singular points of Painlevé equations quite explicitly, which will be discussed in Section 3.

The contents of the paper have been already reported in [T3]. Although we discuss only the Painleve Hamiltonian systems in this paper, it can be proved without any difficulty that singular-perturbative reduction to Birkhoff normal form is possible for any Hamiltonian system. As is explained in [T3], WKB solutions of second-order linear ordinary differential equations can be constructed also through reduction of Hamiltonian systems to Birkhoff normal form. Thus the construction of formal solutions proposed in this paper should be regarded as a natural generalization of that of WKB solutions to Hamiltonian 
systems of singular perturbations.

The author would like to express his gratitude to Professors T. Kawai and T. Aoki for the stimulating discussions with them. He also thanks to Professor M. Yoshino for his valuable comment on Birkhoff normal form. This work is supported by Grant-in-Aid for Scientific Research for Encouragement of Young Scientists (No. 09740101), the Japanese Ministry of Education, Science, Sports and Culture.

\section{§2. Construction of Instanton-Type Formal Solutions via Reduction to Birkhoff Normal Form}

First of all, let us list up Painlevé equations $\left(P_{J}\right)(J=\mathrm{I}, \cdots, \mathrm{VI})$ with a large parameter $\eta$ in Table 1 below.

Table 1.

$$
\begin{aligned}
\left(P_{\mathrm{I}}\right) \quad \frac{d^{2} \lambda}{d t^{2}}= & \eta^{2}\left(6 \lambda^{2}+t\right) . \\
\left(P_{\mathrm{II}}\right) \quad \frac{d^{2} \lambda}{d t^{2}}= & \eta^{2}\left(2 \lambda^{3}+t \lambda+c\right) . \\
\left(P_{\mathrm{III}}\right) \quad \frac{d^{2} \lambda}{d t^{2}}= & \frac{1}{\lambda}\left(\frac{d \lambda}{d t}\right)^{2}-\frac{1}{t} \frac{d \lambda}{d t}+\eta^{2}\left[16 c_{\infty} \lambda^{3}+\frac{8 c_{\infty}^{\prime} \lambda^{2}}{t}-\frac{8 c_{0}^{\prime}}{t}-\frac{16 c_{0}}{\lambda}\right] . \\
\left(P_{\mathrm{IV}}\right) \quad \frac{d^{2} \lambda}{d t^{2}}= & \frac{1}{2 \lambda}\left(\frac{d \lambda}{d t}\right)^{2}-\frac{2}{\lambda}+\eta^{2}\left[\frac{3}{2} \lambda^{3}+4 t \lambda^{2}+\left(2 t^{2}+8 c_{1}\right) \lambda-\frac{8 c_{0}}{\lambda}\right] . \\
\left(P_{\mathrm{V}}\right) \quad \frac{d^{2} \lambda}{d t^{2}}= & \left(\frac{1}{2 \lambda}+\frac{1}{\lambda-1}\right)\left(\frac{d \lambda}{d t}\right)^{2}-\frac{1 d \lambda}{t}+\frac{(\lambda-1)^{2}}{t^{2}}\left(2 \lambda-\frac{1}{2 \lambda}\right) \\
& +\eta^{2} \frac{2 \lambda(\lambda-1)^{2}}{t^{2}}\left[\left(c_{0}+c_{\infty}\right)-\frac{c_{0}}{\lambda^{2}}-\frac{c_{2} t}{(\lambda-1)^{2}}-\frac{c_{1} t^{2}(\lambda+1)}{(\lambda-1)^{3}}\right] . \\
\left(P_{\mathrm{VI}}\right) \quad \frac{d^{2} \lambda}{d t^{2}}= & \frac{1}{2}\left(\frac{1}{\lambda}+\frac{1}{\lambda-1}+\frac{1}{\lambda-t}\right)\left(\frac{d \lambda}{d t}\right)^{2}-\left(\frac{1}{t}+\frac{1}{t-1}+\frac{1}{\lambda-t}\right) \frac{d \lambda}{d t} \\
& +\frac{2 \lambda(\lambda-1)(\lambda-t)}{t^{2}(t-1)^{2}}\left[1-\frac{\lambda^{2}-2 t \lambda+t}{4 \lambda^{2}(\lambda-1)^{2}}\right. \\
& \left.+\eta^{2}\left\{\left(c_{0}+c_{1}+c_{t}+c_{\infty}\right)-\frac{c_{0} t}{\lambda^{2}}+\frac{c_{1}(t-1)}{(\lambda-1)^{2}}-\frac{c_{t} t(t-1)}{(\lambda-t)^{2}}\right\}\right] .
\end{aligned}
$$


As is well known, Painlevé equations can be represented in the form of Painlevé Hamiltonian systems

$$
d \lambda / d t=\eta \partial K_{J} / \partial v, \quad d v / d t=-\eta \partial K_{J} / \partial \lambda
$$

(cf., e.g., [O]). One explicit choice of Hamiltonians $K_{J}(t, \lambda, v, \eta)$ is the following:

\section{Table 2.}

$$
\begin{aligned}
K_{\mathrm{I}}= & \frac{1}{2}\left[v^{2}-\left(4 \lambda^{3}+2 t \lambda\right)\right] . \\
K_{\mathrm{II}}= & \frac{1}{2}\left[v^{2}-\left(\lambda^{4}+t \lambda^{2}+2 c \lambda\right)\right] . \\
K_{\mathrm{III}}= & \frac{2 \lambda^{2}}{t}\left[v^{2}-\eta^{-1} \frac{3 v}{2 \lambda}-\left(\frac{c_{0} t^{2}}{\lambda^{4}}+\frac{c_{0}^{\prime} t}{\lambda^{3}}+\frac{c_{\infty}^{\prime} t}{\lambda}+c_{\infty} t^{2}\right)\right] . \\
K_{\mathrm{IV}}= & 2 \lambda\left[v^{2}-\eta^{-1} \frac{v}{\lambda}-\left(\frac{c_{0}}{\lambda^{2}}+c_{1}+\left(\frac{\lambda+2 t}{4}\right)^{2}\right)\right] . \\
K_{\mathrm{V}}= & \frac{\lambda(\lambda-1)^{2}}{t} \\
& \times\left[v^{2}-\eta^{-1}\left(\frac{1}{\lambda}+\frac{1}{\lambda-1}\right) v-\left(\frac{c_{0}}{\lambda^{2}}+\frac{c_{1} t^{2}}{(\lambda-1)^{4}}+\frac{c_{2} t}{(\lambda-1)^{3}}+\frac{c_{\infty}}{(\lambda-1)^{2}}\right)\right] . \\
K_{\mathrm{VI}}= & \frac{\lambda(\lambda-1)(\lambda-t)}{t(t-1)} \\
& \times\left[v^{2}-\eta^{-1}\left(\frac{1}{\lambda}+\frac{1}{\lambda-1}\right) v-\left(\frac{c_{0}}{\lambda^{2}}+\frac{c_{1}}{(\lambda-1)^{2}}+\frac{c_{\infty}}{\lambda(\lambda-1)}+\frac{c_{t}}{(\lambda-t)^{2}}\right)\right] .
\end{aligned}
$$

In this section we try to construct 2-parameter formal solutions of $\left(P_{J}\right)$ by using reduction of this Hamiltonian system (4) to its Birkhoff normal form.

Let us first note that each Painleve equation has the following structure in common:

$$
\frac{d^{2} \lambda}{d t^{2}}=G_{J}\left(\lambda, \frac{d \lambda}{d t}, t\right)+\eta^{2} F_{J}(\lambda, t)
$$

where $F_{J}$ and $G_{J}$ are rational functions. In view of (5) we easily find that $\left(P_{J}\right)$ has the following formal power series solutions denoted by $\lambda_{J}^{(0)}(t)$ :

$$
\lambda_{J}^{(0)}(t)=\lambda_{0}(t)+\eta^{-2} \lambda_{2}(t)+\eta^{-4} \lambda_{4}(t)+\cdots,
$$


where the top term $\lambda_{0}(t)$ satisfies

$$
F_{J}\left(\lambda_{0}(t), t\right)=0
$$

and the other $\lambda_{2 j}(t)(j \geq 1)$ are determined in a recursive manner. Corresponding to these solutions (6), there exist formal power series solutions called 0-parameter solutions of (4):

$$
\left\{\begin{array}{l}
\lambda_{J}^{(0)}(t)=\lambda_{0}(t)+\eta^{-2} \lambda_{2}(t)+\eta^{-4} \lambda_{4}(t)+\cdots \\
v_{J}^{(0)}(t)=\eta^{-1} v_{1}(t)+\eta^{-3} v_{3}(t)+\eta^{-5} v_{5}(t)+\cdots
\end{array}\right.
$$

(cf. [KT1, Proposition 1.1]). Let us next consider the following localization of (4) at this 0-parameter solution:

$$
\lambda=\lambda_{J}^{(0)}(t)+\eta^{-1 / 2} U, \quad v=v_{J}^{(0)}(t)+\eta^{-1 / 2} V,
$$

that is, we transform the unknown function of $(4)$ from $(\lambda, v)$ to $(U, V)$. Then we readily verify that $(U, V)$ must obey another Hamiltonian system

$$
d U / d t=\eta \partial \mathscr{K}_{J} / \partial V, \quad d V / d t=-\eta \partial \mathscr{K}_{J} / \partial U
$$

where $\mathscr{K}_{J}$ is given by the following:

$$
\mathscr{K}_{J}=\sum_{j+k \geq 2} \eta^{-(j+k-2) / 2} \frac{1}{j ! k !} \frac{\partial^{j+k} K_{J}}{\partial \lambda^{j} \partial v^{k}}\left(t, \lambda_{J}^{(0)}(t), v_{J}^{(0)}(t), \eta\right) U^{j} V^{k}
$$

Now the main result of this paper is the following:

Theorem 1. There exists a formal canonical transformation $(U, V) \mapsto(\tilde{U}, \tilde{V})$ of the form

$$
\left\{\begin{array}{l}
U=u_{0}(\tilde{U}, \tilde{V})+\eta^{-1 / 2} u_{1}(\tilde{U}, \tilde{V})+\cdots \\
V=v_{0}(\tilde{U}, \tilde{V})+\eta^{-1 / 2} v_{1}(\tilde{U}, \tilde{V})+\cdots
\end{array}\right.
$$

where $u_{j}$ and $v_{j}$ are homogeneous polynomials of degree $(j+1)$ in $(\tilde{U}, \tilde{V})$ (whose coefficients are formal power series of $\eta^{-1 / 2}$ with coefficients being functions of t), so that the Hamiltonian system (8) may be taken into the following normal form:

$$
d \tilde{U} / d t=\eta \partial \tilde{\mathscr{K}}_{J} / \partial \tilde{V}, \quad d \tilde{V} / d t=-\eta \partial \tilde{\mathscr{K}}_{J} / \partial \tilde{U}
$$

where

$$
\tilde{\mathscr{K}}_{J}=\sum_{l=0}^{\infty} \eta^{-l} f^{(l)}(t, \eta)(\tilde{U} \tilde{V})^{l+1}
$$

and each $f^{(l)}(t, \eta)=\Sigma_{j \geq 0} \eta^{-j / 2} f_{i / 2}^{(l)}(t)$ is a formal power series of $\eta^{-1 / 2}$ with 
coefficients being functions of $t$.

Remark. The concrete form of the first few terms of $f^{(l)}$ in the case of $J=\mathbb{I}$ is the following:

$$
\begin{aligned}
& f^{(0)}=\left(12 \lambda_{0}\right)^{1 / 2}-\eta^{-2} \frac{3^{2} \cdot 5^{2}}{2}\left(12 \lambda_{0}\right)^{-9 / 2}+\cdots \\
& f^{(1)}=15\left(12 \lambda_{0}\right)^{-2}+\eta^{-2} 3^{3} \cdot 5^{2} \cdot 31\left(12 \lambda_{0}\right)^{-7}+\cdots \\
& f^{(2)}=-3 \cdot 5 \cdot 47\left(12 \lambda_{0}\right)^{-9 / 2}+\cdots
\end{aligned}
$$

For the top degree part $f_{0}^{(0)}(t)$ we also have the following equalities for any $J$ (cf. (27) and (36) below):

$$
f_{0}^{(0)}(t)=\sqrt{\frac{\partial F_{J}}{\partial \lambda}\left(\lambda_{0}(t), t\right)} .
$$

Theorem 1 claims that the Hamiltonian system (8) can be transformed into its Birkhoff normal form. Since the reduced Hamiltonian $\tilde{\mathscr{K}}_{J}$ depends only on the product $\tilde{U} \tilde{V}$, the following relation holds for the system (11):

$$
\frac{d}{d t}(\tilde{U} \tilde{V})=0
$$

Taking this relation (14) into account, we can easily solve the system (11). As a matter of fact,

$$
\left\{\begin{array}{l}
\tilde{U}=\alpha \exp \left(\eta \int^{t} \sum \eta^{-l}(l+1) f^{(l)}(s, \eta)(-\alpha \beta)^{l} d s\right) \\
\tilde{V}=-\beta \exp \left(-\eta \int^{t} \sum \eta^{-l}(l+1) f^{(l)}(s, \eta)(-\alpha \beta)^{l} d s\right)
\end{array}\right.
$$

gives a solution of (11). Subsituting (15) into (10) and then into (7), we obtain 2-parameter formal solutions of $(4)$ and $\left(P_{J}\right)$. The formal solutions of $\left(P_{J}\right)$ thus constructed are sometimes called instanton-type solutions and have the same form with those constructed through the multiple-scale analysis in [AKT2].

Let us now prove Theorem 1. The proof consists of the following two steps; reduction of the linear part and that of the nonlinear part. 


\section{§2.1. Reduction of the Linear Part}

We first seek for a linear canonical transformation

$$
\left\{\begin{array}{l}
U=a(t, \eta) \tilde{U}+b(t, \eta) \tilde{V} \\
V=c(t, \eta) \tilde{U}+d(t, \eta) \tilde{V}
\end{array}\right.
$$

which transforms the Hamiltonian system (8) into its Birkhoff normal form up to quadratic terms. Here $a(t, \eta)$ etc. are formal power series of $\eta^{-1}$. To guarantee that (16) is canonical, we suppose

$$
a(t, \eta) d(t, \eta)-b(t, \eta) c(t, \eta)=1 .
$$

The transformation (16) is obtained also by using the following generating function $W(t, \tilde{U}, V)$ :

$$
W(t, \tilde{U}, V)=-\frac{b}{2 d} V^{2}+\frac{c}{2 d} \tilde{U}^{2}-\frac{1}{d} \tilde{U} V
$$

in other words, (16) is equivalent to

$$
U=-\partial W / \partial V, \quad \tilde{V}=-\partial W / \partial \tilde{U}
$$

By (16) the Hamiltonian $\mathscr{K}_{J}$ is transformed into $\tilde{\mathscr{K}}_{J}$ which is described in terms of the generating function $W$ as follows:

$$
\begin{aligned}
\tilde{\mathscr{K}}_{J}= & \mathscr{K}_{J}(t, U(\tilde{U}, \tilde{V}), V(\tilde{U}, \tilde{V}))+\eta^{-1} \frac{\partial W}{\partial t}(t, \tilde{U}, V(\tilde{U}, \tilde{V})) \\
= & \frac{1}{2} \frac{\partial^{2} K}{\partial \lambda^{2}}(a \tilde{U}+b \tilde{V})^{2}+\frac{\partial^{2} K}{\partial \lambda \partial v}(a \tilde{U}+b \tilde{V})(c \tilde{U}+d \tilde{V})+\frac{1}{2} \frac{\partial^{2} K}{\partial v^{2}}(c \tilde{U}+d \tilde{V})^{2} \\
& +\eta^{-1}\left\{-\left(\frac{b}{2 d}\right)^{\prime}(c \tilde{U}+d \tilde{V})^{2}+\left(\frac{c}{2 d}\right)^{\prime} \tilde{U}^{2}-\left(\frac{1}{d}\right)^{\prime} \tilde{U}(c \tilde{U}+d \tilde{V})\right\} \\
& +(\text { terms of degree greater than } 2 \text { in }(\tilde{U}, \tilde{V})),
\end{aligned}
$$

where' denotes the differentiation with respect to $t$. (Here and in what follows we often omit the suffix $J$ for simplicity and abbreviate $\left(\partial^{2} K_{J} / \partial \lambda^{2}\right)\left(t, \lambda_{J}^{(0)}(t)\right.$, $\left.v_{J}^{(0)}(t), \eta\right)$ to $\partial^{2} K / \partial \lambda^{2}$ etc. if there is no fear of confusions.) Namely

(20) (coeff. of $\tilde{U} \tilde{V}$ in $\left.\tilde{\mathscr{K}}_{J}\right)=\frac{\partial^{2} K}{\partial \lambda^{2}} a b+\frac{\partial^{2} K}{\partial \lambda \partial v}(a d+b c)+\frac{\partial^{2} K}{\partial v^{2}} c d$ 


$$
-\eta^{-1}\left(\left(\frac{b}{d}\right)^{\prime} c d+\left(\frac{1}{d}\right)^{\prime} d\right)
$$

(21) (coeff. of $\tilde{U}^{2}$ in $\left.\tilde{\mathscr{K}}_{J}\right)=\frac{1}{2} \frac{\partial^{2} K}{\partial \lambda^{2}} a^{2}+\frac{\partial^{2} K}{\partial \lambda \partial v} a c+\frac{1}{2} \frac{\partial^{2} K}{\partial v^{2}} c^{2}$

$$
+\eta^{-1}\left(-\left(\frac{b}{2 d}\right)^{\prime} c^{2}+\left(\frac{c}{2 d}\right)^{\prime}-\left(\frac{1}{d}\right)^{\prime} c\right)
$$

(22) (coeff. of $\tilde{V}^{2}$ in $\left.\tilde{\mathscr{K}}_{J}\right)=\frac{1}{2} \frac{\partial^{2} K}{\partial \lambda^{2}} b^{2}+\frac{\partial^{2} K}{\partial \lambda \partial \nu} b d+\frac{1}{2} \frac{\partial^{2} K}{\partial v^{2}} d^{2}-\eta^{-1}\left(\frac{b}{2 d}\right)^{\prime} d^{2}$.

We are thus required to choose $a, b, c$ and $d$ so that (21) and (22) may vanish. It is really possible, that is, we can prove

Proposition 1 . There exist $a, b, c$ and $d$ which sastisfy

$$
\begin{aligned}
& a d-b c=1, \\
& \text { (coeff. of } \tilde{U}^{2} \text { in } \tilde{\mathscr{K}}_{J} \text { ) }=0, \\
& \text { (coeff. of } \tilde{V}^{2} \text { in } \tilde{\mathscr{K}}_{J} \text { ) }=0
\end{aligned}
$$

together with the additional requirement

$$
a=-b .
$$

These conditions (23)-(26) determine $a, b, c$ and $d$ (almost) uniquely. Furthermore (23)-(26) entail the following:

$$
\text { (coeff. of } \tilde{U} \tilde{V} \text { in } \tilde{\mathscr{K}}_{J} \text { ) }=\eta^{-1} S_{\text {odd }}
$$

where $S_{\mathrm{odd}}$ denotes the odd part (in the sense of [AKT2, Definition 2.1]) of solutions of the Riccati equation associated with the Fréchet derivative (i.e., linearized equation) of (4) along the 0-parameter solution $\left(\lambda_{J}^{(0)}, v_{J}^{(0)}\right)$.

Remark. It is obvious that (23)-(25) cannot determine the transformation uniquely. To determine $a, b, c$ and $d$ we make the additional requirement (26) in Proposition 1. The meaning of (26) is to pick out the odd part of solutions as the coefficient of $\tilde{U} \tilde{V}$ (i.e., (27)) and the even part as the canonical transformation $a$ and $b$ (cf. (42) below). 
Before proving Proposition 1, let us recall here the definition of the Riccati equation associated with the Fréchet derivative of (4).

Substituting $\lambda=\lambda_{J}^{(0)}+\psi$ and $v=v_{J}^{(0)}+\varphi$ into (4), we find that the Fréchet derivative of (4) is given by the following:

$$
\left\{\begin{array}{l}
\psi^{\prime}=\eta\left(\frac{\partial^{2} K}{\partial \lambda \partial \nu} \psi+\frac{\partial^{2} K}{\partial v^{2}} \varphi\right), \\
\varphi^{\prime}=-\eta\left(\frac{\partial^{2} K}{\partial \lambda^{2}} \psi+\frac{\partial^{2} K}{\partial \lambda \partial \nu} \varphi\right) .
\end{array}\right.
$$

We consider WKB solutions of (28), which is of the form

$$
\psi=\exp \int^{t} S d t, \quad \varphi=\exp \int^{t} T d t
$$

Then $S$ and $T$ must satisfy

$$
\begin{aligned}
& \left(S-\eta \frac{\partial^{2} K}{\partial \lambda \partial v}\right) \exp \int^{t} S d t-\eta \frac{\partial^{2} K}{\partial v^{2}} \exp \int^{t} T d t=0 \\
& \eta \frac{\partial^{2} K}{\partial \lambda^{2}} \exp \int^{t} S d t+\left(T+\eta \frac{\partial^{2} K}{\partial \lambda \partial v}\right) \exp \int^{t} T d t=0
\end{aligned}
$$

Let us take the logarithmic derivative of (29).

$$
\frac{d}{d t} \log \left(S-\eta \frac{\partial^{2} K}{\partial \lambda \partial v}\right)+S=\frac{d}{d t} \log \frac{\partial^{2} K}{\partial v^{2}}+T .
$$

Furthermore, since neither $\exp \int{ }^{t} S d t$ nor $\exp \int{ }^{t} T d t$ is equal to zero, (29) and (30) imply

$$
\left(S-\eta \frac{\partial^{2} K}{\partial \lambda \partial v}\right)\left(T+\eta \frac{\partial^{2} K}{\partial \lambda \partial v}\right)+\eta^{2} \frac{\partial^{2} K}{\partial \lambda^{2}} \frac{\partial^{2} K}{\partial v^{2}}=0
$$

A single equation which determines $S$ can be easily obtained from (31) and (32). In fact, putting

$$
S^{\dagger}=S-\eta \frac{\partial^{2} K}{\partial \lambda \partial \nu}, \quad T^{\dagger}=T+\eta \frac{\partial^{2} K}{\partial \lambda \partial v},
$$


we have

$$
\begin{aligned}
& T^{\dagger}=S^{\dagger}+\frac{d}{d t} \log S^{\dagger}+2 \eta \frac{\partial^{2} K}{\partial \lambda \partial v}-\frac{d}{d t} \log \frac{\partial^{2} K}{\partial v^{2}} \\
& S^{\dagger} T^{\dagger}+\eta^{2} \frac{\partial^{2} K}{\partial \lambda^{2}} \frac{\partial^{2} K}{\partial v^{2}}=0 .
\end{aligned}
$$

Hence

$$
\left(S^{\dagger}\right)^{2}+\frac{d S^{\dagger}}{d t}+\left(2 \eta \frac{\partial^{2} K}{\partial \lambda \partial v}-\frac{d}{d t} \log \frac{\partial^{2} K}{\partial v^{2}}\right) S^{\dagger}+\eta^{2} \frac{\partial^{2} K}{\partial \lambda^{2}} \frac{\partial^{2} K}{\partial v^{2}}=0
$$

or, in terms of the original $S$ instead of $S^{\dagger}$,

$$
\begin{aligned}
& S^{2}+\frac{d S}{d t}-\frac{d}{d t} \log \frac{\partial^{2} K}{\partial v^{2}}+\eta^{2}\left(\frac{\partial^{2} K}{\partial \lambda^{2}} \frac{\partial^{2} K}{\partial v^{2}}-\left(\frac{\partial^{2} K}{\partial \lambda \partial v}\right)^{2}\right) \\
& +\eta\left(\frac{\partial^{2} K}{\partial \lambda \partial v} \frac{d}{d t} \log \frac{\partial^{2} K}{\partial v^{2}}-\frac{d}{d t} \frac{\partial^{2} K}{\partial \lambda \partial v}\right)=0
\end{aligned}
$$

should be satisfied. This is the Riccati equation associated with the Fréchet derivative of (4).

We can solve (33) in a singular-perturbative manner to obtain two formal power series solutions

$$
\begin{aligned}
S_{ \pm} & = \pm \eta S_{-1}(t)+S_{ \pm, 0}(t)+\eta^{-1} S_{ \pm, 1}(t)+\cdots \\
& = \pm S_{\text {odd }}+S_{\text {even }}
\end{aligned}
$$

Note that comparison of the odd part (in the sense of [AKT2, Definition 2.1]) of (33) entails the following:

$$
S_{\text {even }}=\frac{1}{2} \frac{d}{d t}\left(\log \frac{\partial^{2} K}{\partial v^{2}}-\log S_{\text {odd }}\right) .
$$

Furthermore, since the degree 0 part (in $\eta$ ) of $\left(\partial^{2} K / \partial \lambda \partial v\right)$ vanishes, by straightforward computations we can show the following:

$$
S_{-1}(t)=\left(\frac{\partial F_{J}}{\partial \lambda}\left(\lambda_{0}, t\right)\right)^{1 / 2}
$$

(cf. (1.11) in [KT1], (1.35) and (1.41) in [KT2]). 
Proof of Proposition 1. Let us note that

$$
\begin{aligned}
(25) \Leftrightarrow & \frac{\partial^{2} K}{\partial \lambda^{2}}+2 \frac{\partial^{2} K}{\partial \lambda \partial v} \frac{d}{b}+\frac{\partial^{2} K}{\partial v^{2}}\left(\frac{d}{b}\right)^{2}-\eta^{-1}\left(\frac{b}{d}\right)^{\prime}\left(\frac{d}{b}\right)^{2}=0 \\
\Leftrightarrow & \frac{\partial^{2} K}{\partial \lambda^{2}}+2 \frac{\partial^{2} K}{\partial \lambda \partial v} \frac{d}{b}+\frac{\partial^{2} K}{\partial v^{2}}\left(\frac{d}{b}\right)^{2}+\eta^{-1}\left(\frac{d}{b}\right)^{\prime}=0 \\
\Leftrightarrow & \left(\eta \frac{\partial^{2} K}{\partial v^{2}} \frac{d}{b}\right)^{2}+\left(\eta \frac{\partial^{2} K}{\partial v^{2}} \frac{d}{b}\right)^{\prime} \\
& +\left(2 \eta \frac{\partial^{2} K}{\partial \lambda \partial v}-\frac{d}{d t} \log \frac{\partial^{2} K}{\partial v^{2}}\right) \eta \frac{\partial^{2} K}{\partial v^{2}} \frac{d}{b}+\eta^{2} \frac{\partial^{2} K}{\partial \lambda^{2}} \frac{\partial^{2} K}{\partial v^{2}}=0
\end{aligned}
$$

Hence $\eta \frac{\partial^{2} K}{\partial v^{2}} \frac{d}{b}+\eta \frac{\partial^{2} K}{\partial \lambda \partial v}$ is a solution of the Riccati equation (33). Similarly, since (23) implies $(b / d)^{\prime}=(a / c)^{\prime}-(1 / c d)^{\prime}$, we have

$$
\begin{aligned}
(24) \Leftrightarrow & \frac{\partial^{2} K}{\partial \lambda^{2}} a^{2}+2 \frac{\partial^{2} K}{\partial \lambda \partial v} a c+\frac{\partial^{2} K}{\partial v^{2}} c^{2} \\
& +\eta^{-1}\left(-\left(\frac{a}{c}\right)^{\prime} c^{2}+\left(\frac{1}{c d}\right)^{\prime} c^{2}+\left(\frac{c}{d}\right)^{\prime}-2\left(\frac{1}{d}\right)^{\prime} c\right)=0 \\
\Leftrightarrow & \frac{\partial^{2} K}{\partial \lambda^{2}} a^{2}+2 \frac{\partial^{2} K}{\partial \lambda \partial v} a c+\frac{\partial^{2} K}{\partial v^{2}} c^{2}-\eta^{-1}\left(\frac{a}{c}\right)^{\prime} c^{2}=0 \\
\Leftrightarrow & \left.\frac{\partial^{2} K}{\partial \lambda^{2}}+2 \frac{\partial^{2} K}{\partial \lambda \partial v} \frac{\partial^{2} K}{a}+\frac{c}{\partial v^{2}} \frac{\frac{c}{a}}{a}\right)^{2}+\eta^{-1}\left(\frac{c}{a}\right)^{\prime}=0 \\
\Leftrightarrow & \left(\eta \frac{\partial^{2} K}{\partial v^{2}} \frac{c}{a}\right)^{2}+\left(\eta \frac{\partial^{2} K c}{\partial v^{2}} \frac{1}{a}\right)^{\prime} \\
& +\left(2 \eta \frac{\partial^{2} K}{\partial \lambda \partial v}-\frac{d}{d t} \log \frac{\partial^{2} K}{\partial v^{2}}\right) \eta \frac{\partial^{2} K c}{\partial v^{2}} \frac{-}{a}+\eta^{2} \frac{\partial^{2} K}{\partial \lambda^{2}} \frac{\partial^{2} K}{\partial v^{2}}=0 .
\end{aligned}
$$

That is, $\eta \frac{\partial^{2} K c}{\partial v^{2}} \frac{-}{a}+\eta \frac{\partial^{2} K}{\partial \lambda \partial v}$ is also a solution of (33). In view of (23) they cannot be the same solutions. Thus we may assume 


$$
\left\{\begin{array}{l}
\eta \frac{\partial^{2} K}{\partial v^{2}} \frac{c}{a}+\eta \frac{\partial^{2} K}{\partial \lambda \partial v}=S_{\mathrm{odd}}+S_{\mathrm{even}}=S_{+} \\
\eta \frac{\partial^{2} K}{\partial v^{2}} \frac{d}{b}+\eta \frac{\partial^{2} K}{\partial \lambda \partial v}=-S_{\mathrm{odd}}+S_{\mathrm{even}}=S_{-}
\end{array}\right.
$$

and

$$
\eta \frac{\partial^{2} K}{\partial v^{2}} \frac{1}{a b}=\eta \frac{\partial^{2} K}{\partial v^{2}}\left(\frac{d}{b}-\frac{c}{a}\right)=-2 S_{\mathrm{odd}}
$$

These relations (38) and (39) together with the additional requirement (26) determine $a, b, c$ and $d$ almost uniquely (i.e., up to the choice of the branch of $S_{-1}$ ). In particular,

$$
a=-b=\left(\frac{\partial^{2} K}{\partial v^{2}} \frac{1}{2 \eta^{-1} S_{\mathrm{odd}}}\right)^{1 / 2} .
$$

Furthermore, it follows from (37), $a d-b c=1$ and the identity

$$
\begin{aligned}
-\left(\frac{b}{d}\right)^{\prime} c d-\left(\frac{1}{d}\right)^{\prime} d & =\left(\frac{d}{b}\right)^{\prime} \frac{b^{2} c}{d}+\frac{d^{\prime}}{d} \\
& =\left(\frac{d}{b}\right)^{\prime} \frac{b}{d}(a d-1)+\frac{d^{\prime}}{d} \\
& =\left(\frac{d}{b}\right)^{\prime} a b-\left(\frac{d}{b}\right)^{\prime} \frac{b}{d}+\frac{d^{\prime}}{d} \\
& =\left(\frac{d}{b}\right)^{\prime} a b+\frac{b^{\prime}}{b}
\end{aligned}
$$

that the coefficient of $\tilde{U} \tilde{V}$ becomes

$$
\begin{aligned}
& \frac{\partial^{2} K}{\partial \lambda^{2}} a b+\frac{\partial^{2} K}{\partial \lambda \partial v}(a d+b c)+\frac{\partial^{2} K}{\partial v^{2}} c d-\eta^{-1}\left(\left(\frac{b}{d}\right)^{\prime} c d+\left(\frac{1}{d}\right)^{\prime} d\right) \\
& =\frac{\partial^{2} K}{\partial \lambda^{2}} a b+\frac{\partial^{2} K}{\partial \lambda \partial v}(a d+b c)+\frac{\partial^{2} K}{\partial v^{2}} c d+\eta^{-1}\left(\left(\frac{d}{b}\right)^{\prime} a b+\frac{b^{\prime}}{b}\right) \\
& =-\left(2 \frac{\partial^{2} K}{\partial \lambda \partial v} \frac{d}{b}+\frac{\partial^{2} K}{\partial v^{2}}\left(\frac{d}{b}\right)^{2}\right) a b+\frac{\partial^{2} K}{\partial \lambda} \frac{\partial v}{\partial v}(a d+b c)+\frac{\partial^{2} K}{\partial v^{2}} c d+\eta^{-1} \frac{b^{\prime}}{b}
\end{aligned}
$$




$$
\begin{aligned}
& =\frac{\partial^{2} K}{\partial \lambda \partial v}(-a d+b c)+\frac{\partial^{2} K}{\partial v^{2}}\left(-\frac{a d^{2}}{b}+c d\right)+\eta^{-1} \frac{b^{\prime}}{b} \\
& =-\frac{\partial^{2} K}{\partial \lambda \partial v}-\frac{\partial^{2} K}{\partial v^{2}} \frac{d}{b}+\eta^{-1} \frac{b^{\prime}}{b} \\
& =-\eta^{-1} S_{-}+\eta^{-1} \frac{b^{\prime}}{b} .
\end{aligned}
$$

Since (35) and (40) imply

$$
\frac{d}{d t} \log b=\frac{1}{2}\left(\frac{d}{d t} \log \frac{\partial^{2} K}{\partial v^{2}}-\frac{d}{d t} \log S_{\text {odd }}\right)=S_{\text {even }},
$$

we consequently have

$$
\text { (coeff. of } \begin{aligned}
\tilde{U} \tilde{V}) & =-\eta^{-1}\left(-S_{\text {odd }}+S_{\text {even }}\right)+\eta^{-1} S_{\text {even }} \\
& =\eta^{-1} S_{\text {odd }} .
\end{aligned}
$$

Q.E.D.

Summing up, as reduction of the linear part we have obtained a linear canonical transformation (16) with the following coefficients:

$$
\begin{aligned}
& a=-b=\left(\frac{\partial^{2} K}{\partial v^{2}} \frac{1}{2 \eta^{-1} S_{\mathrm{odd}}}\right)^{1 / 2}, \\
& c=\left(2 \eta^{-1} S_{\mathrm{odd}} \frac{\partial^{2} K}{\partial v^{2}}\right)^{-1 / 2}\left(\eta^{-1} S_{+}-\frac{\partial^{2} K}{\partial \lambda \partial \nu}\right) \\
& d=-\left(2 \eta^{-1} S_{\mathrm{odd}} \frac{\partial^{2} K}{\partial \nu^{2}}\right)^{-1 / 2}\left(\eta^{-1} S_{-}-\frac{\partial^{2} K}{\partial \lambda \partial v}\right)
\end{aligned}
$$

By this linear transformation the Hamiltonian is reduced to

(46) $\tilde{\mathscr{K}}_{J}=\eta^{-1} S_{\text {odd }} \tilde{U} \tilde{V}+\sum_{j+k \geq 3} \eta^{-(j+k-2) / 2} \frac{1}{j ! k !} \frac{\partial^{j+k} K_{J}}{\partial \lambda^{j} \partial v^{k}}(a \tilde{U}+b \tilde{V})^{j}(c \tilde{U}+d \tilde{V})^{k}$

\section{§2.2. Reduction of the Nonlinear Part}

By the reduction of the linear part explained in Subsection 2.1 we have 
obtained the following reduced Hamiltonian:

$$
\begin{aligned}
\mathscr{K}_{J} & =f^{(0)} U V+\sum_{j+k \geq 3} \epsilon^{j+k-2} \frac{1}{j ! k !} \frac{\partial^{j+k} \mathbb{K}}{\partial \lambda^{j} \partial \nu^{k}}(a U+b V)^{j}(c U+d V)^{k} \\
& =f^{(0)} U V+\sum_{|\vec{j}+\vec{k}| \geq 3} \epsilon^{|\vec{j}+\vec{k}|-2} \frac{1}{\vec{j} ! \vec{k} !} \frac{\partial^{|\vec{j}+\vec{k}|} K}{\partial \lambda^{|\vec{j}|} \partial v^{|\vec{k}|}} a^{j_{1}} b^{j_{2}} c^{k_{1}} d^{k_{2}} U^{j_{1}+k_{1}} V^{j_{2}+k_{2}},
\end{aligned}
$$

(cf. (46)) where $\vec{j}=\left(j_{1}, j_{2}\right)$ and $\vec{k}=\left(k_{1}, k_{2}\right)$. Here and throughout this subsection $\eta^{-1 / 2}$ is denoted by $\epsilon$ and $\epsilon^{2} S_{\text {odd }}$ is abbreviated by $f^{(0)}$. (We have omitted tildes $(\sim)$ in (46) for the sake of simplicity.) The Hamiltonian (47) is written also in the following form:

$$
\mathscr{K}_{J}=f^{(0)} U V+\sum_{\substack{p+q \geq 1 \\ p, q \geq-1}} \epsilon^{p+q} K_{p q}(t, \epsilon) U^{p+1} V^{q+1}
$$

where

$$
K_{p q}(t, \epsilon)=\sum_{\substack{j_{1}+k_{1}=p+1 \\ j_{2}+k_{2}=q+1}} \frac{1}{\vec{j} ! k !} \frac{\partial^{|\vec{j}+\vec{k}|} K}{\partial \lambda^{|\vec{j}|} \partial v^{|\vec{k}|}} a^{j_{1}} b^{j_{2}} c^{k_{1}} d^{k_{2}}
$$

In this subsection we consider reduction of the nonlinear part, that is, we try to find a canonical transformation with the trivial linear terms

$$
\left\{\begin{array}{l}
U=\tilde{U}+\epsilon u_{1}(t, \tilde{U}, \tilde{V}, \epsilon)+\epsilon^{2} u_{2}(t, \tilde{U}, \tilde{V}, \epsilon)+\cdots \\
V=\tilde{V}+\epsilon v_{1}(t, \tilde{U}, \tilde{V}, \epsilon)+\epsilon^{2} v_{2}(t, \tilde{U}, \tilde{V}, \epsilon)+\cdots
\end{array}\right.
$$

where

$$
\begin{aligned}
& u_{j}(t, \tilde{U}, \tilde{V}, \epsilon)=\sum_{\substack{p+q=j-1 \\
p, q \geq-1}} u_{p q}(t, \epsilon) \tilde{U}^{p+1} \tilde{V}^{q+1} \\
& v_{j}(t, \tilde{U}, \tilde{V}, \epsilon)=\sum_{\substack{p+q=j-1 \\
p, q \geq-1}} v_{p q}(t, \epsilon) \tilde{U}^{p+1} \tilde{V}^{q+1}
\end{aligned}
$$

with $u_{p q}$ and $v_{p q}$ being formal power series of $\epsilon$, which transforms the Hamiltonian $\mathscr{K}_{J}$ into its Birkhoff normal form.

For that purpose we again make use of a generating function of the following form: 


$$
\begin{aligned}
W & =W(t, \tilde{U}, V) \\
& =-\tilde{U} V+\sum_{\substack{p+q \geq 1 \\
p, q \geq-1}} \epsilon^{p+q} a_{p q}(t, \epsilon) \tilde{U}^{p+1} V^{q+1} .
\end{aligned}
$$

In what follows we try to determine $\left\{a_{p q}\right\}$ so that the associated canonical transformation

$$
\left\{\begin{array}{l}
U=-\frac{\partial W}{\partial V}=\tilde{U}-\sum_{\substack{p+q \geq 1 \\
p, q \geq-1}} \epsilon^{p+q}(q+1) a_{p q} \tilde{U}^{p+1} V^{q} \\
\tilde{V}=-\frac{\partial W}{\partial \tilde{U}}=V-\sum_{\substack{p+q \geq 1 \\
p, q \geq-1}} \epsilon^{p+q}(p+1) a_{p q} \tilde{U}^{p} V^{q+1}
\end{array}\right.
$$

reduces the Hamiltonian (48) into its Birkhoff normal form. If we successfully find such $\left\{a_{p q}\right\}$, then $u_{j}(t, \tilde{U}, \tilde{V}, \epsilon)$ and $v_{j}(t, \tilde{U}, \tilde{V}, \epsilon)$ are explicitly given in the following way: First let us substitute $V=\tilde{V}+\epsilon v_{1}+\epsilon^{2} v_{2}+\cdots$ into the second equation of (54) and compare the coefficients of like powers of $\epsilon$, then we find

$$
v_{j}=\sum_{\substack{p+q+\mu_{1}+\ldots+\mu_{k+1}=j \\ p+q \geq 1, p, q \geq-1, \mu_{l} \geq 0}}(p+1) a_{p q} \tilde{U}^{p} v_{\mu_{1}} \cdots v_{\mu_{k+1}}
$$

$(j=1,2,3, \cdots)$ where $v_{0}$ denotes $\tilde{V}$. Next let us substitute the same expansion for $V$ into the first equation of (54), then we obtain

$$
u_{j}=-\sum_{\substack{p+q+\mu_{1}+\cdots+\mu_{k}=j \\ p+q \geq 1, p . q \geq-1, \mu_{l} \geq 0}}(q+1) a_{p q} \tilde{U}^{p+1} v_{\mu_{1}} \cdots v_{\mu_{k}}
$$

$(j=1,2,3, \cdots)$. (We conventionally define $u_{0}=\tilde{U}$.) The relations $(55)$ and (56) recursively determine $\left\{u_{j}\right\}$ and $\left\{v_{j}\right\}$ from $\left\{a_{p q}\right\}$. For example,

$$
\begin{aligned}
& v_{1}=3 a_{2-1} \tilde{U}^{2}+2 a_{10} \tilde{U} \tilde{V}+a_{01} \tilde{V}^{2}, \\
& u_{1}=-\left(a_{10} \tilde{U}^{2}+2 a_{01} \tilde{U} \tilde{V}+3 a_{-12} \tilde{V}^{2}\right) .
\end{aligned}
$$

In general, we can prove the following by an induction. 


\section{Lemma 1.}

$$
\left\{\begin{array}{l}
v_{j}=\sum_{\substack{p+q=j \\
p, q \geq-1}}(p+1) a_{p q} \tilde{U}^{p} \tilde{V}^{q+1}+R_{j} \\
u_{j}=\sum_{\substack{p+q=j \\
p, q \geq-1}}(q+1) a_{p q} \tilde{U}^{p+1} \tilde{V}^{q}+\tilde{R}_{j}
\end{array}\right.
$$

where $R_{j}$ and $\tilde{R}_{j}$ are some polynomials of $\left\{a_{p q}\right\}_{p+q \leq j-1}$.

Now let us show how $\left\{a_{p q}\right\}$ should be determined. Substituting the expansion (50), we find that (50) transforms the Hamiltonian $\mathscr{K}_{J}$ into

$$
\begin{aligned}
\widetilde{\mathscr{K}}_{J}= & \mathscr{K}_{J}+\epsilon^{2} \frac{\partial W}{\partial t} \\
= & f^{(0)}\left(\sum_{j \geq 0} \epsilon^{j} u_{j}\right)\left(\sum_{j \geq 0} \epsilon^{j} v_{j}\right) \\
& +\sum_{\substack{p+q \geq 1 \\
p, q \geq-1}} \epsilon^{p+q} K_{p q}\left(\sum_{j \geq 0} \epsilon^{j} u_{j}\right)^{p+1}\left(\sum_{j \geq 0} \epsilon^{j} v_{j}\right)^{q+1} \\
& +\epsilon^{2} \sum_{\substack{p+q \geq 1 \\
p, q \geq-1}} \epsilon^{p+q} \frac{\partial a_{p q}}{\partial t} \tilde{U}^{p+1}\left(\sum_{j \geq 0} \epsilon^{j} v_{j}\right)^{q+1} .
\end{aligned}
$$

Here $\left\{u_{j}\right\}$ and $\left\{v_{j}\right\}$ are expressed in terms of $\left\{a_{p q}\right\}$ through the relations (55) and (56). In particular, the degree $l$ part $(\operatorname{in}(\tilde{U}, \tilde{V}))$ of $\tilde{\mathscr{K}}_{J}$ has the following form:

$$
\begin{aligned}
& \tilde{K}_{J, l}=f^{(0)} \epsilon^{l-2} \sum_{j+k=l-2} u_{j} v_{k} \\
&+\epsilon^{l-2} \sum_{\substack{p+q+\mu_{1}+\cdots+\mu_{p++1}+v_{1}+\cdots+v_{q+1}=l-2 \\
p+q \geq 1, p, q \geq-1, \mu_{l}, v_{l} \geq 0}} K_{p q} u_{\mu_{1}} \cdots u_{\mu_{p+1}} v_{v_{1}} \cdots v_{v_{q+1}} \\
&+\epsilon_{\substack{p+q+v_{1}+\ldots+v_{q+1}=l-2 \\
p+q \geq 1, p, q \geq-1, v_{l} \geq 0}} \frac{\partial a_{p q}}{\partial t} \tilde{U}^{p+1} v_{v_{1}} \cdots v_{v_{p+1}} .
\end{aligned}
$$

Let $\mathscr{K}^{(1)}$ (resp., $\mathscr{K}^{(2)}, \mathscr{K}^{(3)}$ ) denote the first (resp., second, third) term of the right-hand side of (59). Then it follows from Lemma 1 that 
(coeff. of $\tilde{U}^{j+1} \tilde{V}^{k+1}$ in $\left.\mathscr{K}^{(1)}\right)=f^{(0)} \epsilon^{l-2}(j-k) a_{j k}+\mathscr{R}^{(1)}$,

where $j+k=l-2$ and $\mathscr{R}^{(1)}$ is a polynomial of $\left\{a_{p q}\right\}_{p+q \leq l-3}$. Similarly, we can show

$$
\begin{aligned}
& \text { (coeff. of } \left.\tilde{U}^{j+1} \tilde{V}^{k+1} \text { in } \mathscr{K}^{(2)}\right)=\mathscr{R}^{(2)}, \\
& \text { (coeff. of } \left.\tilde{U}^{j+1} \tilde{V}^{k+1} \text { in } \mathscr{K}^{(3)}\right)=\epsilon^{l} \frac{\partial a_{j k}}{\partial t}+\mathscr{R}^{(3)},
\end{aligned}
$$

where $\mathscr{R}^{(2)}$ and $\mathscr{R}^{(3)}$ are also polynomials of $\left\{a_{p q}\right\}_{p+q \leq l-3}$ (and of their first derivatives with respect to $t$ ). Hence we have

\section{Lemma 2.}

$$
\text { (coeff. of } \left.\tilde{U}^{j+1} \tilde{V}^{k+1} \text { in } \tilde{\mathscr{K}}_{J}\right)=f^{(0)} \epsilon^{l-2}(j-k) a_{j k}+\epsilon^{l} \frac{\partial a_{j k}}{\partial t}+\epsilon^{l-2} \mathscr{R}_{j k}
$$

where $j+k=l-2$ and $\mathscr{R}_{j k}$ is a polynomial of $\left\{a_{p q}\right\}_{p+q \leq l-3}$ and of their first derivatives with respect to $t$.

In order that $\tilde{\mathscr{K}}_{J}$ is of Birkhoff normal form, we have to require

$$
\text { (coeff. of } \tilde{U}^{j+1} \tilde{V}^{k+1} \text { in } \tilde{\mathscr{K}}_{J} \text { ) }=0
$$

for any $j, k$ satisfying $j \neq k$. Note that, when $j \neq k$, this relation (61) can be solved for $\left\{a_{j k}\right\}(j \neq k)$ in a singular-perturbative manner thanks to Lemma 2 . Thus $\left\{a_{j k}\right\}$ except $\left\{a_{j j}\right\}$ are uniquely determined by the relations (61) in a recursive way and they actually transform $\mathscr{K}_{J}$ into its Birkhoff normal form.

At this stage $\left\{a_{j j}\right\}$ are not still fixed. To determine them we introduce, in addition to (26), more additional requirements in the following way: The canonical transformation obtained so far produces 2-parameter instanton-type formal solutions of $\left(P_{J}\right)$ of the form

$$
\lambda_{J}^{(0)}+\eta^{-1 / 2}\left(\frac{\partial^{2} K}{\partial v^{2}} \frac{1}{2 \eta^{-1} S_{\text {odd }}}\right)^{1 / 2} \sum_{j \geq 0} \eta^{-j / 2}\left(u_{j}(t, \tilde{U}, \tilde{V}, \eta)-v_{j}(t, \tilde{U}, \tilde{V}, \eta)\right)
$$

where $\tilde{U}$ and $\tilde{V}$ are given by (15). In particular, we look at the degree $(2 l+1)$ part (in $(\tilde{U}, \tilde{V})$ ) of $(62)$, which is represented as

$$
\eta^{-(2 l+1) / 2}\left(\frac{\partial^{2} K}{\partial v^{2}} \frac{1}{2 \eta^{-1} S_{\text {odd }}}\right)^{1 / 2}\left(u_{2 l}-v_{2 l}\right)
$$




$$
\begin{gathered}
=\eta^{-(2 l+1) / 2}\left(\frac{\partial^{2} K}{\partial v^{2}} \frac{1}{2 \eta^{-1} S_{\text {odd }}}\right)^{1 / 2} \sum_{\substack{j+k=2 l-1 \\
j . k \geq-1}} g_{j k}(t, \eta) \tilde{U}^{j+1} \tilde{V}^{k+1} \\
=\eta^{-(2 l+1) / 2}\left(\frac{\partial^{2} K}{\partial v^{2}} \frac{1}{2 \eta^{-1} S_{\text {odd }}}\right)^{1 / 2} \sum_{\substack{j+k=2 l-1 \\
j, k \geq-1}} g_{j k}(t, \eta) \alpha^{j+1}(-\beta)^{k+1} \\
\quad \times \exp \left((j-k) \eta \int^{t} \sum \eta^{-l}(l+1) f^{(l)}(-\alpha \beta)^{l} d s\right) .
\end{gathered}
$$

Among these terms the following two terms

$$
\eta^{-(2 l+1) / 2}\left(\frac{\partial^{2} K}{\partial v^{2}} \frac{1}{2 \eta^{-1} S_{\text {odd }}}\right)^{1 / 2} g_{l+1, l} \alpha^{l+2}(-\beta)^{l+1} \exp \left(\eta \int^{t} \cdots d s\right)
$$

and

$$
\eta^{-(2 l+1) / 2}\left(\frac{\partial^{2} K}{\partial v^{2}} \frac{1}{2 \eta^{-1} S_{\text {odd }}}\right)^{1 / 2} g_{l . l+1} \alpha^{l+1}(-\beta)^{l+2} \exp \left(-\eta \int^{t} \cdots d s\right)
$$

(" $( \pm 1)$-instanton terms") are important in the sense that WKB solutions of the Fréchet derivative of $\left(P_{J}\right)$ contribute only to them. Taking this fact into account, we make the following additional requirement

$$
g_{l+1, l}=-g_{l, l+1}
$$

for any $l$ with $l \geq 1$ as a generalization of (26). Since Lemma 1 entails

$$
\left\{\begin{array}{l}
g_{l+1, l}=-(l+1) a_{l l}-(l+2) a_{l+1 l-1}+\tilde{g}_{l+1, l} \\
g_{l, l+1}=-(l+1) a_{l l}-(l+2) a_{l-1 l+1}+\tilde{g}_{l, l+1}
\end{array}\right.
$$

where $\tilde{g}_{l+1, l}$ and $\tilde{g}_{l . l+1}$ are polynomials of $\left\{a_{j k}\right\}_{j+k \leq 2 l-1}$, the requirement (63) becomes

$$
a_{l l}=\frac{l+2}{2(l+1)}\left(a_{l+1 l-1}+a_{l-1 l+1}\right)+\frac{\tilde{g}_{l+1 . l}+\tilde{g}_{l . l+1}}{2(l+1)}
$$

and uniquely determines $\left\{a_{l l}\right\}$ from $\left\{a_{j k}\right\}(j \neq k)$ in a recursive manner. This completes the proof of Theorem 1. 


\section{§3. Local Behavior of Instanton-Type Formal Solutions of $\left(P_{J}\right)$ near Regular-Type Singular Points}

We have seen in the preceding section that singular-perturbative reduction of (4) (more precisely, its localization at the 0-parameter solution) to Birkhoff normal form produces 2-parameter formal solutions of $\left(P_{J}\right)$. Looking at this construction of 2-parameter solutions more carefully, we try to analyze their local behavior at fixed regular-type singular points in this section. As a typical example of fixed regular-type singular points of Painlevé equations we pick up the origin $t=0$ of the sixth Painleve equation $\left(P_{\mathrm{VI}}\right)$ and discuss the problem only for this typical example in this paper.

As is shown in Theorem 2 below, the regular-type singularness of fixed singular points of $\left(P_{J}\right)\left(t=0\right.$ of $\left(P_{\mathrm{VI}}\right)$ here) should entail the simpleness of poles which the coefficients $f^{(l)}(t, \eta)$ of the Birkhoff normal form may possess there. Furthermore, in the global study of $\left(P_{J}\right)$ the residues of $f^{(l)}(t, \eta)$ at regular-type singular points would play an important role. Hence it is desirable to be able to compute such residues explicitly. However, our choice of Hamiltonians $K_{J}(t, \lambda, v, \eta)$ which is listed up in Table 2 is not convenient for that purpose; if we work with $K_{J}$, we can show the simpleness of poles, but the computation of the residues becomes quite difficult. To overcome this difficulty we use the following "polynomial Hamiltonian $H_{\mathrm{VI}}$ "

$$
d \lambda / d t=\eta \partial H_{\mathrm{VI}} / \partial \mu, \quad d \mu / d t=-\eta \partial H_{\mathrm{VI}} / \partial \lambda
$$

where

$$
\begin{aligned}
H_{\mathrm{VI}}= & \frac{1}{t(t-1)}\left[\lambda(\lambda-1)(\lambda-t) \mu^{2}-\eta^{-1}\left\{\kappa_{0}(\lambda-1)(\lambda-t)+\kappa_{1} \lambda(\lambda-t)\right.\right. \\
& \left.\left.+\left(\kappa_{t}-1\right) \lambda(\lambda-1)\right\} \mu+\frac{1}{4} \eta^{-2}\left\{\left(\kappa_{0}+\kappa_{1}+\kappa_{t}-1\right)^{2}-\kappa_{\infty}^{2}\right\}(\lambda-t)\right],
\end{aligned}
$$

which is first discovered by Okamoto ([O]), instead of $K_{\mathrm{VI}}$ in this paper. (Roughly speaking, by using $H_{\mathrm{VI}}$ we can "triangularize" the problem of computing the residues in a sense (cf. (90) and (91) below).) The relations between $H_{\mathrm{vI}}, \mu$, $\kappa_{*}$ and $K_{\mathrm{VI}}, v, c_{*}$ are given by the following:

$$
\begin{aligned}
& \frac{1}{4}\left(\kappa_{*}^{2}-1\right)=c_{*} \eta^{2} \quad \text { where } *=0,1, t, \\
& \frac{1}{4}\left(\kappa_{\infty}^{2}-\kappa_{0}^{2}-\kappa_{1}^{2}-\kappa_{t}^{2}-1\right)=c_{\infty} \eta^{2},
\end{aligned}
$$




$$
\begin{aligned}
& \mu+\frac{1}{2} \eta^{-1}\left(\frac{1-\kappa_{0}}{\lambda}+\frac{1-\kappa_{1}}{\lambda-1}+\frac{1-\kappa_{t}}{\lambda-t}\right)=v, \\
& H_{\mathrm{VI}}+\frac{1}{2} \eta^{-2}\left(1-\kappa_{t}\right)\left(\frac{1-\kappa_{0}}{t}+\frac{1-\kappa_{1}}{t-1}+\frac{1}{\lambda-t}\right)=K_{\mathrm{VI}}
\end{aligned}
$$

Note that every $\kappa_{*}(*=0,1, t, \infty)$ is a quantity of degree 1 in $\eta$.

Let us now state our result. The top degree part $\lambda_{0}(t)$ of our formal solutions is characterized by the equation $F_{\mathrm{VI}}\left(\lambda_{0}(t), t\right)=0$, i.e.,

$$
\left(c_{0}+c_{1}+c_{t}+c_{\infty}\right)-c_{0} \frac{t}{\lambda_{0}^{2}}+c_{1} \frac{t-1}{\left(\lambda_{0}-1\right)^{2}}-c_{t} \frac{t(t-1)}{\left(\lambda_{0}-t\right)^{2}}=0 .
$$

This algebraic equation has six solutions, one of which shows the following behavior at $t=0$ :

$$
\lambda_{0}(t)=a t+b t^{2}+\cdots \quad \text { with } a=\frac{\sqrt{c_{0}}}{\sqrt{c_{0}}+\sqrt{c_{t}}} .
$$

We restrict ourselves to this special choice of $\lambda_{0}(t)$ in this paper. (The other cases will be discussed elsewhere.) Then, for 2-parameter formal solutions with the above top degree part $\lambda_{0}(t)$, we can verify the following:

Theorem 2. Let $f^{(l)}(t, \eta)$ be the coefficients of the Birkhoff normal form obtained in Theorem 1 from the localization of (66) at the 0-parameter solution with the top degree part $\lambda_{0}$ satisfying (68). Then each $f^{(l)}(t, \eta)$ has a simple pole at $t=0$ and

$$
\begin{aligned}
& \operatorname{Res}_{t=0}^{(0)}(t, \eta)=\eta^{-1}\left(\kappa_{0}+\kappa_{t}\right) \\
& \operatorname{Res}_{t=0}^{(1)}(t, \eta)=1, \\
& \operatorname{Res}_{t=0}^{(l)}(t, \eta)=0 \quad(l \geq 2) .
\end{aligned}
$$

Proof. During the proof we often omit the suffix VI. Let us first investigate the local behavior of the 0 -parameter solution $\left(\lambda^{(0)}, \mu^{(0)}\right)=\left(\Sigma \eta^{-j} \lambda_{j}(t)\right.$, 
$\left.\Sigma \eta^{-j} \mu_{j}(t)\right)$ at $t=0$. (Note that $\lambda_{j}(t)$ identically vanishes for every odd integer $j$. Similarly $v_{j}(t)$ vanishes for every even integer $j$, but this is not true for $\mu_{j}(t)$.) In view of $(68)$ and the explicit form of $\left(P_{\mathrm{vl}}\right)$ (cf. Table 1) we find

$$
\begin{aligned}
& \frac{\partial^{j} F}{\partial \lambda^{j}}\left(\lambda_{0}, t\right)=\mathcal{O}\left(t^{-(j+1)}\right) \quad \text { for any } j \geq 1, \\
& \lambda=\mathscr{O}(t) \Rightarrow G_{\mathrm{Vl}}\left(\lambda, \frac{d \lambda}{d t}, t\right)=\mathcal{O}\left(t^{-1}\right)
\end{aligned}
$$

(where $(\mathcal{O}$ denotes Landau's symbol). Making use of these properties, we can verify the following by an induction:

$$
\lambda_{j}(t)=\mathcal{O}(t) \quad \text { for any } j \geq 0 \text {. }
$$

Then the first equation of the Hamiltonian system (66), i.e.,

$$
\begin{aligned}
& 2 \lambda^{(0)}\left(\lambda^{(0)}-1\right)\left(\lambda^{(0)}-t\right) \mu^{(0)}=\eta^{-1} t(t-1) \frac{d \lambda^{(0)}}{d t} \\
& \quad+\eta^{-1}\left[\kappa_{0}\left(\lambda^{(0)}-1\right)\left(\lambda^{(0)}-t\right)+\kappa_{1} \lambda^{(0)}\left(\lambda^{(0)}-t\right)+\left(\kappa_{t}-1\right) \lambda^{(0)}\left(\lambda^{(0)}-1\right)\right],
\end{aligned}
$$

entails

$$
\mu_{j}(t)=\mathcal{O}\left(t^{-1}\right) \quad \text { for any } j \geq 0
$$

Furthermore, we can compute the coefficients of the top degree part in $t$ of $\left(\lambda^{(0)}, \mu^{(0)}\right)$, which is denoted by $(A, B)$ in what follows, explicitly. (Of course, $A$ and $B$ are formal power series of $\eta^{-1}$.) As a matter of fact, comparing the top degree part in $t$ of the Hamiltonian system (66), we obtain

$$
\left\{\begin{array}{l}
A=2 \eta A(A-1) B-\left(\kappa_{0}+\kappa_{t}-1\right) A+\kappa_{0}, \\
B=\eta(2 A-1) B^{2}-\left(\kappa_{0}+\kappa_{t}-1\right) B
\end{array}\right.
$$

This algebraic equation (74) for $A$ and $B$ has the following two solutions:

$$
(A, B)=\left(\frac{\kappa_{0}}{\kappa_{0}+\kappa_{t}}, 0\right) \quad \text { or } \quad\left(\frac{\kappa_{0}}{\kappa_{0}-\kappa_{t}}, \eta^{-1}\left(\kappa_{0}-\kappa_{t}\right)\right) .
$$

Comparison of the top degree part of $A$ in $\eta^{-1}$ with (68) tells us that the first one gives a true answer in our case. We have thus verified 
Lemma 3. The 0-parameter solution $\left(\lambda^{(0)}, \mu^{(0)}\right)$ with the top degree part $\lambda_{0}$ satisfying (68) has the following expansion at $t=0$ :

$$
\lambda^{(0)}=A t+\cdots, \quad \mu^{(0)}=\frac{B}{t}+\cdots
$$

with

$$
A=\frac{\kappa_{0}}{\kappa_{0}+\kappa_{t}}, \quad B=0
$$

Lemma 3 and a straightforward computation also show

Lemma 4. We abbreviate $\left(\partial^{j+k} H_{\mathrm{vl}} / \partial \lambda^{j} \partial \mu^{k}\right)\left(t, \lambda^{(0)}, \mu^{(0)}, \eta\right)$ to $\partial^{j+k} H / \partial \lambda^{j} \partial \mu^{k}$. Then we have

$$
\begin{aligned}
& \frac{\partial^{j+2} H}{\partial \lambda^{j} \partial \frac{H}{\partial \mu^{2}}}=\left\{\begin{array}{lc}
2 A(A-1) t+\cdots & \text { for } j=0 \\
2(2 A-1)+\cdots & \text { for } j=1 \\
\frac{4}{t}+\cdots & \text { for } j=2 \\
-\frac{12}{t}+\cdots & \text { for } j=3
\end{array}\right. \\
& \frac{\partial^{j+k} H}{\partial \lambda^{j} \partial \mu^{k}}=\left\{\begin{array}{lll}
\mathcal{O}(1) & \text { for } j=0 \text { and } k=0,1 \\
\mathcal{O}\left(t^{-1}\right) & \text { for } j=1,2,3 \text { and } k=0,1 .
\end{array}\right.
\end{aligned}
$$

Furthermore

$$
\operatorname{Res}_{t=0} \partial^{2} H / \partial \lambda \partial \mu=-\eta^{-1}\left(\kappa_{0}+\kappa_{t}-1\right)
$$

Let us next consider the linear canonical transformation, which reduces the linear part of (66) to Birkhoff normal form, obtained in Section 2.1. Note that all formulas in Section 2 are valid even for the polynomial Hamiltonian $H_{\mathrm{VI}}$, if we replace $K_{\mathrm{VI}}$ and $v$ by $H_{\mathrm{VI}}$ and $\mu$ respectively. For example, the Riccati equation associated with the Fréchet derivative of (66) is given by the following: 
(77) $S^{2}+\frac{d S}{d t}-\frac{d}{d t} \log \frac{\partial^{2} H}{\partial \mu^{2}}$

$$
+\eta^{2}\left\{\frac{\partial^{2} H}{\partial \lambda^{2}} \frac{\partial^{2} H}{\partial \mu^{2}}-\left(\frac{\partial^{2} H}{\partial \lambda \partial \mu}\right)^{2}+\eta^{-1} \frac{\partial^{2} H}{\partial \lambda \partial \mu} \frac{d}{d t} \log \frac{\partial^{2} H}{\partial \mu^{2}}-\eta^{-1} \frac{d}{d t} \frac{\partial^{2} H}{\partial \lambda \partial \mu}\right\}=0 .
$$

It follows from Lemma 4 that

$$
\begin{aligned}
& -\frac{d}{d t} \log \frac{\partial^{2} H}{\partial \mu^{2}}=-\frac{1}{t}+\cdots \\
& \frac{\partial^{2} H}{\partial \lambda^{2}} \frac{\partial^{2} H}{\partial \mu^{2}}-\cdots-\eta^{-1} \frac{d}{d t} \frac{\partial^{2} H}{\partial \lambda \partial \mu}=-\eta^{-2}\left(\left(\kappa_{0}+\kappa_{t}\right)^{2}-1\right) \frac{1}{t^{2}}+\cdots
\end{aligned}
$$

Hence, by a similar argument as in the proof of [AKT1, Proposition 3.1], we can verify

Proposition 2. The formal power series solutions $S_{ \pm}$of (77) have a simple pole at $t=0$ and their residues are given by

$$
\operatorname{Res}_{t=0} S_{ \pm}=1 \pm\left(\kappa_{0}+\kappa_{t}\right)
$$

The relation (69) is an immediate consequence of (27) and (78). The following local behavior at $t=0$ of the coefficients of the linear canonical transformation also follows from Proposition 2 and (43)-(45):

$$
\begin{aligned}
& a=-b=\left(\frac{-\kappa_{0} \kappa_{t}}{\eta^{-1}\left(\kappa_{0}+\kappa_{t}\right)^{3}}\right)^{1 / 2} t+\cdots \\
& c=\left(\frac{\eta^{-1}\left(\kappa_{0}+\kappa_{t}\right)^{3}}{-\kappa_{0} \kappa_{t}}\right)^{1 / 2} \frac{1}{t}+\cdots \\
& d=0 \cdot \frac{1}{t}+\cdots=\emptyset(1) .
\end{aligned}
$$

Let us now discuss the nonlinear part of the canonical transformation, which reduces 


$$
\mathscr{H}_{\mathrm{VI}}=f^{(0)} U V+\sum_{\substack{p+q \geq 1 \\ p . q \geq-1}} \eta^{-(p+q) / 2} H_{p q}(t, \eta) U^{p+1} V^{q+1}
$$

with

$$
H_{p q}(t, \eta)=\sum_{\substack{j_{1}+k_{1}=p+1 \\ j_{2}+k_{2}=q+1}} \overline{\frac{j}{j ! \vec{k} !}} \frac{\partial^{|\vec{j}+\vec{k}|} H}{\partial \lambda^{|\vec{j}|} \partial \mu^{|\vec{k}|}} a^{j_{1}} b^{j_{2}} c^{k_{1}} d^{k_{2}}
$$

to its Birkhoff normal form, obtained in Section 2.2. By Lemma 4 and (79)-(81) we readily find

$$
H_{p q}=\mathcal{O}\left(t^{-1}\right)
$$

for any $p, q$ and further

(85) only $H_{3-1}, H_{20}, H_{11}, H_{2-1}$ and $H_{10}$ have a simple pole at $t=0$.

Let us recall that the coefficients $\left\{a_{p q}\right\}$ of the generating function (53) of transformation are uniquely determined by the requirements (61) and (65). Hence, in view of (84), we can prove the following by an induction.

Proposition 3. (i) The coefficients $\left\{a_{p q}\right\}$ of the generating function are holomorphic (more precisely, formal power series of $\eta^{-1 / 2}$ with holomorphic coefficients) at $t=0$ for any $p, q$ with $p, q \geq-1$ and $p+q \geq 1$.

(ii) The coefficients $f^{(l)}(t, \eta)$ of the Birkhoff normal form shows the following behavior at $t=0$ :

$$
f^{(l)}=\mathcal{O}\left(t^{-1}\right) \quad \text { for any } l \geq 1
$$

We finally compute the residues of $f^{(l)}(t, \eta)$. A key idea is to use the relation between the two Hamiltonians

$$
\tilde{\mathscr{H}}=\mathscr{H}+\eta^{-1} \frac{\partial W}{\partial t}
$$

or,

$$
\tilde{\mathscr{H}}\left(t, \tilde{U},-\frac{\partial W}{\partial \tilde{U}}\right)=\mathscr{H}\left(t,-\frac{\partial W}{\partial V}, V\right)+\eta^{-1} \frac{\partial W}{\partial t}(t, \tilde{U}, V)
$$

Our determination of $\left\{a_{p q}\right\}$ entails that (88) identically holds for any $(\tilde{U}, V)$. 
(Otherwise stated, (88) is an equality as formal power series of $(\tilde{U}, V)$.) Note that both sides of (88) has an at most simple pole at $t=0$ thanks to Proposition 3. In particular, the second term of the right-hand side is holomorphic there. Therefore, comparing the residues at $t=0$ of both sides of (88), we obtain

$$
\begin{aligned}
\hat{f}^{(0)} & \sum_{\substack{p+q \geq 1 \\
p, q \geq-1}} \eta^{-(p+q) / 2}(p-q) \hat{a}_{p q} \tilde{U}^{p+1} V^{q+1} \\
= & \sum_{l \geq 1} \eta^{-l} \hat{f}^{(l)} \tilde{U}^{l+1}\left(V-\sum_{\substack{p+q \geq 1 \\
p, q \geq-1}} \eta^{-(p+q) / 2}(p+1) \hat{a}_{p q} \tilde{U}^{p} V^{q+1}\right)^{l+1} \\
& -\sum_{\substack{\mu+v \geq 1 \\
\mu, v \geq-1}} \eta^{-(\mu+v) / 2} \hat{H}_{\mu \nu}\left(\tilde{U}-\sum_{\substack{p+q \geq 1 \\
p, q \geq-1}} \eta^{-(p+q) / 2}(q+1) \hat{a}_{p q} \tilde{U}^{p+1} V^{q}\right)^{\mu+1} V^{v+1},
\end{aligned}
$$

where $\hat{f}^{(l)}, \hat{H}_{p q}$ and $\hat{a}_{p q}$ respectively denote $\operatorname{Res}_{t=0} f^{(l)}, \operatorname{Res}_{t=0} H_{p q}$ and $\left.a_{p q}\right|_{t=0}$. For example, comparison of cubic terms (with respect to $(\tilde{U}, V)$ ) of both sides of (89) deduces

$$
\begin{aligned}
& 3 \hat{f}^{(0)} \hat{a}_{2-1}=-\hat{H}_{2-1}, \quad \hat{f}^{(0)} \hat{a}_{10}=-\hat{H}_{10}, \\
& -\hat{f}^{(0)} \hat{a}_{01}=-\widehat{H}_{01}, \quad-3 \hat{f}^{(0)} \hat{a}_{-12}=-\widehat{H}_{-12} .
\end{aligned}
$$

Since $\hat{H}_{01}=\widehat{H}_{-12}=0$ by $(85)$, we consequently have $\hat{a}_{01}=\hat{a}_{-12}=0$. More generally,

\section{Lemma 5.}

$$
\hat{a}_{p q}=0 \quad \text { for } p<q
$$

Lemma 5 can be proved by an induction in the following way: We compare the coefficients of $\tilde{U}^{p+1} V^{q+1}(p<q)$ of both sides of (89). Then from the left-hand side

$$
\hat{f}^{(0)} \eta^{-(p+q) / 2}(p-q) \hat{a}_{p q}
$$

appears, while there is no contribution from the right-hand side because of the induction hypothesis and the following relation

$$
\widehat{H}_{p q}=0 \quad \text { for } p<q
$$

(which immediately follows from (85)). Thus we have (90). 
To compute the residues of $f^{(l)}(t, \eta)$, we compare the coefficients of $\tilde{U}^{j+1} V^{j+1}$ $(j \geq 1)$ of both sides of $(89)$. The coefficient of the left-hand side is trivially equal to 0 . On the other hand, taking Lemma 5 into account, we find that for the right-hand side the contributions come only from

$$
\begin{aligned}
\sum_{l \geq 1} \eta^{-l} \hat{f}^{(l)}(\tilde{U} V)^{l+1}\left(1-\sum_{p \geq 1} \eta^{-p}(p+1) \hat{a}_{p p} \tilde{U}^{p} V^{p}\right)^{l+1} \\
\quad-\sum_{\mu \geq 1} \eta^{-\mu} \hat{H}_{\mu \mu}(\tilde{U} V)^{\mu+1}\left(1-\sum_{p \geq 1} \eta^{-p}(p+1) \hat{a}_{p p} \tilde{U}^{p} V^{p}\right)^{\mu+1} .
\end{aligned}
$$

Hence the coefficient of the right-hand side is

$$
\eta^{-j} \sum_{\substack{l+m=j \\ l \geq 1, m \geq 0}}(-1)^{l+1}\left(\hat{f}^{(l)}-\widehat{H}_{l l}\right) \sum_{p_{1}+\cdots+p_{l+1}=m}\left(p_{1}+1\right) \cdots\left(p_{l+1}+1\right) \hat{a}_{p_{1} p_{1}} \cdots \hat{a}_{p_{l+1} p_{l+1}}
$$

(where we define $\hat{a}_{00}=-1$ for the sake of convention). Since all $\widehat{H}_{l l}$ vanishes except for $l=1$ (cf. (85)), we obtain

$$
\begin{aligned}
& \sum_{\substack{l+m=j \\
l \geq 1, m \geq 0}}(-1)^{l+1} \hat{f}^{(l)} \sum_{p_{1}+\cdots+p_{l+1}=m}\left(p_{1}+1\right) \cdots\left(p_{l+1}+1\right) \hat{a}_{p_{1} p_{1}} \cdots \hat{a}_{p_{l+1} p_{l+1}} \\
& \quad=\widehat{H}_{11} \sum_{p+q=j-1}(p+1)(q+1) \hat{a}_{p p} \hat{a}_{q q} .
\end{aligned}
$$

For $j=1$ the relation (92) becomes

$$
\hat{f}^{(1)}=\widehat{H}_{11}
$$

and for $j \geq 2$ (92) implies the following:

$$
\hat{f}^{(j)}=\sum_{\substack{l+m=j \\ l \geq 2, m \geq 1}}(-1)^{l+1} \hat{f}^{(l)} \sum_{p_{1}+\cdots+p_{l+1}=m}\left(p_{1}+1\right) \cdots\left(p_{l+1}+1\right) \hat{a}_{p_{1} p_{1}} \cdots \hat{a}_{p_{l+1} p_{l+1}} .
$$

Here it follows from (79), (80), (83) and Lemma 4 that

$$
\widehat{H}_{11}=\operatorname{Res}_{t=0} H_{11}=\frac{1}{4} \operatorname{Res}\left(\frac{\partial^{4} H}{\partial \lambda^{2} \partial \mu^{2}} a^{2} c^{2}\right)=1 \text {. }
$$

Thus we have verified 


$$
\hat{f}^{(1)}=1 \quad \text { and } \quad \hat{f}^{(j)}=0(j \geq 2)
$$

This completes the proof of Theorem 2.

Q.E.D.

\section{References}

[AKT1] Aoki, T., Kawai, T. and Takei, Y., Algebraic analysis of singular perturbations, Sūgaku Expositions, 8 (1995), 217-240. (Originally appeared in Japanese in Sügaku, 45 (1993), 299-315.)

[AKT2] W - WKB analysis of Painlevé transcendents with a large parameter. II, Structure of Solutions of Differential Equations, World Scientific, 1996, pp. 1-49.

[B] Birkhoff, G. D., Dynamical Systems, Amer. Math. Soc., 1927, revised edition, 1966.

[KT1] Kawai, T. and Takei, Y., WKB analysis of Painlevé transcendents with a large parameter. I, Adv. Math., 118 (1996), 1-33.

[KT2] WKB analysis of Painlevé transcendents with a large parameter. III, $A d v$. Math., 134 (1998), 178-218.

[O] Okamoto, K., Isomonodromic deformation and Painlevé equations, and the Garnier systems, J. Fac. Sci. Univ. Tokyo, Sect. IA, 33 (1986), 575-618.

[SM] Siegel, C. L. and Moser, J. K., Lectures on Celestial Mechanics, Springer-Verlag, 1971.

[T1] Takei, Y., On a WKB-theoretic approach to the Painlevé transcendents, XIth International Congress of Mathematical Physics, International Press, 1995, pp. 533-542.

[T2] - On a WKB-theoretic approach to the Painleve transcendents. II, RIMS Kôkyûroku, 1058 (1998), 114-128.

[T3] — Birkhoff normal form of Hamiltonian systems and WKB-type formal solutions, to appear in RIMS Kôkyûroku "Resurgent Functions and Convolution Equatins".

[Tka1] Takano, K., Reduction for Painlevé equations at the fixed singular points of the first kind, Funkcial. Ekvac., 29 (1986), 99-119.

[Tka2] - Reduction for Painlevé equations at the fixed singular points of the second kind, J. Math. Soc. Japan, 42 (1990), 423-443. 
